\title{
Pathway for surgeons and programs to establish and maintain a successful robot-assisted adult cardiac surgery program
}

\author{
Evelio Rodriguez, MD, ${ }^{\mathrm{a}}$ L. Wiley Nifong, MD, ${ }^{\mathrm{b}}$ Johannes Bonatti, MD, FETCS, ${ }^{\mathrm{c}}$ Roberto Casula, FRCS, ${ }^{\mathrm{d}}$ \\ Volkmar Falk, MD, ${ }^{\mathrm{e}}$ Thierry A. Folliguet, MD, ${ }^{\mathrm{f}}$ Bob B. Kiaii, MD ${ }^{\mathrm{g}}$ Michael J. Mack, MD, ${ }^{\mathrm{h}}$ \\ Tomislav Mihaljevic, MD, ${ }^{\mathrm{i}}$ J. Michael Smith, MD, ${ }_{\mathrm{j}}$ Rakesh M. Suri, MD, DPhil, ${ }^{\mathrm{k}}$ Joseph E. Bavaria, MD, \\ Thomas E. MacGillivray, MD, ${ }^{\mathrm{m}}$ and W. Randolph Chitwood, Jr, $\mathrm{MD}^{\mathrm{n}}$
}

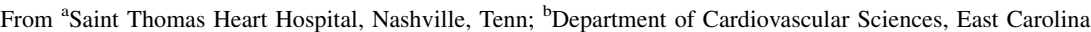
Heart Institute, Greenville, NC; ${ }^{~}$ Cleveland Clinic Abu Dhabi Heart and Vascular Institute, United Arab Emirates; ' Imperial College Healthcare NHS Trust and Department of Cardiothoracic Surgery, St. Mary's Hospital, London, United Kingdom; ${ }^{\mathrm{e} D e u t s c h e s ~ H e r z z e n t r u m ~ B e r l i n, ~ C h a r i t e ~ B e r l i n, ~ B e r l i n, ~ G e r m a n y ; ~}{ }^{\mathrm{f}}$ Centre Hospitalo-Universitaire Brabois ILCV, Vandoeuvre les Nancy, France; ${ }^{\mathrm{g}}$ Department of Cardiovascular and Thoracic Surgery, London Health Sciences Centre, London, Ontario, Canada; ${ }^{\mathrm{h}}$ Baylor Health Care System, The Heart Hospital, Baylor, Plano, Tex; ${ }^{i}$ Cleveland Clinic Abu Dhabi, United Arab Emirates; ${ }^{j}$ Good Samaritan Hospital, Cincinnati, Ohio; ${ }^{\mathrm{k}} \mathrm{Cleveland}$ Clinic Foundation, Cleveland, Ohio; ${ }^{\mathrm{l} D i v i s i o n}$ of Cardiovascular Surgery, Hospital of the University of Pennsylvania, Philadelphia, Pa; ${ }^{\mathrm{m}}$ Massachusetts General Hospital, Boston, Mass; and ${ }^{\mathrm{n}}$ Vidant Medical Center and East Carolina University, Greenville, NC.

Disclosures: Authors have nothing to disclose with regard to commercial support.

This article has been co-published with permission in The Journal of Thoracic and Cardiovascular Surgery and The Annals of Thoracic Surgery.

Received for publication Nov 30, 2015; revisions received Nov 30, 2015; accepted for publication Feb 18, 2016. Address for reprints: Evelio Rodriguez, MD, Saint Thomas Heart Hospital, 4230 Harding Rd, Ste 430, Nashville,

TN 37205 (E-mail: evelio.rodriguez@sth.org).

J Thorac Cardiovasc Surg 2016;152:9-13

$0022-5223 / \$ 36.00$

Copyright (C) 2016 by The Society of Thoracic Surgeons

http://dx.doi.org/10.1016/j.jtcvs.2016.05.018
\end{abstract}

\section{DEFINITION}

Robotic cardiac surgery is defined as any cardiac operation that is performed either completely or in part using robotassisted (ie, telemanipulation) technology. At present, for robot-assisted cardiac operations, the Food and Drug Administration (FDA) has approved only one device, the da Vinci surgical system (Intuitive Surgical, Sunnyvale, Calif).

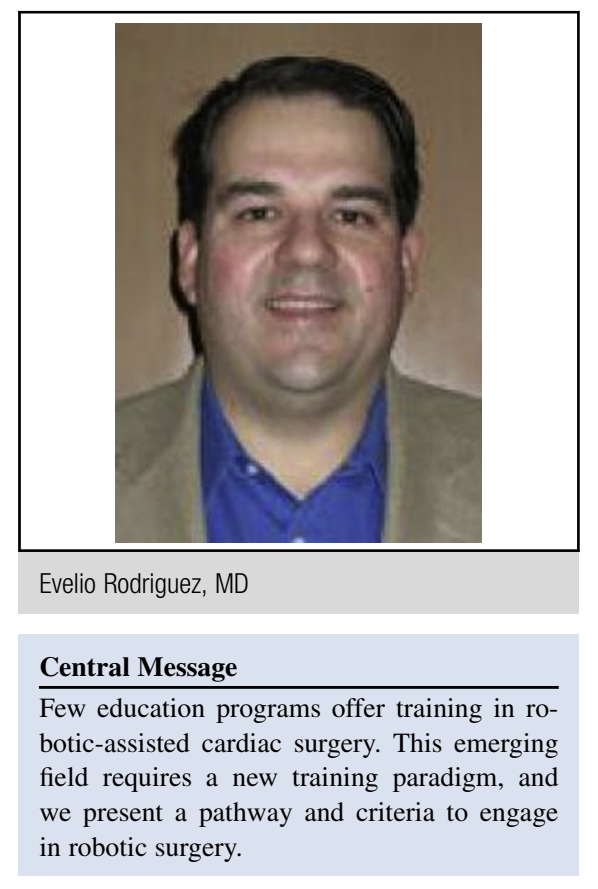

See Editorial Commentary page 14.

\section{PREAMBLE}

The da Vinci robotic surgical system was FDA approved for mitral valve surgery in 2002 after phase I and phase II investigative device exemption trials. ${ }^{1,2}$ In 2004, the system was FDA approved for coronary revascularization. ${ }^{3}$ Since then, there has been slow adoption of this technology in cardiac surgery for many reasons, including (1) cost; (2) technical demands; (3) need for significant institutional resources (ie, dedicated operating room, nursing, anesthesia, and perfusion teams); and (4) more importantly, lack of proper training, and for robotic mitral surgery, the lack of prior experience in mitral valve repair and conventional minimally invasive techniques. That has resulted in only a few centers worldwide being able to establish successful and enduring da Vinci robotic cardiac surgical programs. ${ }^{4-14}$

Cardiac surgeons receive appropriate training for conventional sternotomy-based cardiac surgical operations in either residency or postgraduate programs; however, only a few education programs offer training specifically in minimally invasive cardiac surgery. Moreover, these programs generally do not provide training in robotic-assisted cardiac surgery. For surgeons to participate in this emerging field in a safe and efficacious manner, a new training paradigm has become necessary. Many surgeons desire more guidance from professional organizations when establishing robotic cardiac surgical programs.

The members of this writing group are experienced with robotic-assisted adult cardiac surgery procedures. Although we understand that there are several potential pathways to establish a successful adult robotic-assisted cardiac surgery program, this document presents a potential pathway and set of recommendations for both individual surgeons and surgical programs to begin or to participate in robotic-assisted adult cardiac surgery. By adhering to these criteria, cardiac surgeons can expect to follow minimum safety standards for implementation and independent conduct of robot-assisted cardiac surgical procedures. This document establishes criteria supported by The Society of Thoracic Surgeons (STS) and The American Association for Thoracic Surgery (AATS). It is well recognized that a coordinated surgical team, which includes cardiac anesthesiologists, cardiac 
surgeons, operating room assistants, nurses, and perfusionists, is critical to establish a successful robot-assisted cardiac surgical program.

\section{BACKGROUND}

Currently, robot-assisted telemanipulation is used mainly for two cardiac surgical categories: (1) intracardiac operations (mitral valve, tricuspid valve, atrial septal defect, cardiac tumors, and atrial fibrillation); and (2) coronary revascularization. This document delineates the recommendations from a group of experienced robotic cardiac surgeons for cardiac surgery programs that want to perform these operations safely and independently.

\section{CRITERIA}

\section{Robotic Cardiac Surgical Team Requirements}

Institutional. To establish a successful robotic cardiac surgical program, there must be a strong hospital commitment, as well as commitment from all team members, including cardiac anesthesiologists, cardiologists, cardiac surgeons, perfusionists, and operating room personnel. Individual surgeons should start programs only if this type of full commitment is apparent. In addition, the team must have had substantial cardiac surgical experience as a unit. It cannot be overemphasized that transition from traditional sternotomy-based operations to a minimally invasive robotic-assisted platform requires an experienced and cohesive cardiac surgical team. Hospital administrators and physician leaders should be aware of the necessity of surgeon and team learning curves, which in some complex procedures can reach three digit numbers. Long operative times and technical difficulties, requiring conversion to sternotomy or thoracotomy, should be expected during the implementation phase.

Individual. A single cardiac surgeon should be designated to be the team leader. This person should coordinate the strategic action plan, when developing a robotic cardiac surgical program, as well as monitor program quality and safety. Initially, one main robot-assisted application (ie, an intracardiac procedure versus coronary revascularization) should be chosen based on the surgeon's current practice. Simultaneous introduction of different procedures can be more challenging; however, that can have synergistic effects for reducing the learning curve and can help maintain adequate robotic procedure volumes. Surgeons selecting an intracardiac platform should be experienced in mitral/tricuspid valve operations as well as other open-heart procedures. If coronary revascularization is selected as the initial pathway, the surgeon should have solid coronary revascularization experience, which may include off-pump techniques and, ideally, minithoracotomy coronary revascularization approaches. Larger case volumes of conventional cardiac operations best prepare surgeons and teams for the initiation of a robotic cardiac surgical program.

The anesthesia team must have significant experience with cardiac surgery patients who have comorbidities. They should have significant experience with intraoperative two-dimensional and, ideally, three-dimensional transesophageal echocardiography (TEE), especially for teams involved in robotic mitral valve surgery. In addition, the anesthesiologist should have expertise with single-lung ventilation, using double-lumen intubation and bronchial blockers. To gain maximal early experience, only one or two cardiac anesthesiologists should be involved in the first 20 robotic cardiac operations. It is best that the anesthesiologists or lead cardiac surgeon have prior experience in placing transjugular cannulas for cardiopulmonary perfusion, retrograde coronary sinus cardioplegia administration, and intracardiac pressure monitoring.

If the program does not have anesthesiologists versed in intraoperative TEE, a dedicated cardiologist with experience in intraoperative imaging should perform TEE studies. It is critical that cardiac surgeons and cardiologists involved in the program define, and agree, which patients benefit most from robot-assisted cardiac operations. Ideally, these decisions should be made in the setting of a multidisciplinary team case conference. In addition, they must carefully monitor the intraoperative and postoperative quality outcomes. It is prudent to begin a robotic program by selecting low operative risk patients with simple pathology. That is extremely important and ensures physiologic reserves should learning curve cases become complicated, extensively long, or necessitate conversion to an alternate approach.

The perfusion team and the cardiac surgeon should select one or two perfusionists who will perform the first 20 operations or as many as the lead surgeon determines are necessary to maintain expertise. They should have prior experience in managing peripheral arteriovenous perfusion with vacuum-assisted or kinetic venous drainage.

A small core group of operating room personnel must be dedicated to participate in each of these procedures. Surgical technicians and nurses must become extremely familiar with the robotic system setup, deployment, and intraoperative organization. It is crucial to have a single person (ie, surgeon, physician assistant, or nurse assistant) selected to be the patient-side assistant for these early cases. Close synchrony between the patient-side assistant, surgeon, and scrub personnel is essential for maximizing temporal economy. Thus, at many experienced robotic centers, the same first assistant and scrub personnel perform every one or at least the vast majority of the robotic-assisted cardiac cases.

Owing to the complexity of these procedures, reviewing a preoperative checklist for availability and function of all devices is recommended. Completion of this checklist should be part of the "time-out" preoperative protocol. 


\section{Surgeon Experience Requirements}

Robotic intracardiac operations. Surgeon expertise in performing both routine and high-risk cardiac procedures is essential. The surgeon should have completed an accredited cardiothoracic surgical training program in which the surgical management of valve disease is a focal point. Ideally, this surgeon either should be eligible or certified by the appropriate cardiac surgical board according to his or her practice location. However, we recommend that the lead surgeon must hold an appropriate certification (eg, board certification by the American Board of Thoracic Surgery or by the certifying institution at the country or region in which she or he practices cardiac surgery) to independently practice cardiac surgery.

The console surgeon should be facile in performing sternotomy-based and minimally invasive intracardiac surgical operations. We recommend that before initiating a robot-assisted cardiac program, the lead surgeon should be comfortable performing and have a good track record with the type of intracardiac surgical procedures that will be performed with robotic assistance. For robot-assisted mitral valve operations, the surgeon should be experienced with all aspects of mitral valve operations, including repair and replacement techniques. It cannot be overemphasized that the surgeon must be facile in all aspects of mitral valve surgery, and specifically repair, with an excellent track record, as these procedures will be prolonged during the initial learning curve. Experience with other intracardiac procedures including aortic valve and tricuspid valve surgery, atrial septal defect closures, cardiac tumor extirpations, or atrial fibrillation procedures will enhance the surgeon's ability to start a robotic-assisted program. Nonetheless, a solid mitral valve surgical experience is absolutely necessary for a successful robotic-assisted mitral valve program.

Robotic coronary revascularization operations. Expertise in performing routine, complex, and high-risk coronary revascularization is essential. As above, the surgeon should have completed an accredited cardiothoracic surgical training program in which the surgical management of coronary artery disease is a focal point. Ideally, this surgeon either should be eligible or certified by the appropriate cardiothoracic surgical board. However, we recommend that the lead surgeon must hold an appropriate certification (eg, board certification by the American Board of Thoracic Surgery or by the certifying institution at the country or region in which she or he practices cardiac surgery) to independently practice cardiac surgery.

The console surgeon should be facile in either sternotomy-based or minimally invasive coronary revascularization techniques. To begin a robotic-assisted coronary revascularization program, the lead surgeon must be experienced with coronary revascularization procedures both on and off pump. We recommend the lead surgeon have a solid off-pump coronary revascularization experience and track record if the team intends to perform their roboticassisted revascularization cases using off-pump techniques. However, if the team plans to perform these cases with cardiopulmonary bypass assistance, then off-pump experience is not required.

Cardiopulmonary bypass skills. For either robotic intracardiac or coronary operations, the surgeon must be able to institute and perform both routine and emergent peripheral cardiopulmonary bypass. Robotic coronary operations require peripheral cardiopulmonary bypass, not only when using arrested heart techniques, but also during off-pump revascularization, when immediate conversion to a pump operation becomes necessary. Therefore, we recommend that the lead surgeon be experienced with peripheral cannulation techniques. These may include peripheral cannulations (femoral or axillary arteries; femoral or jugular veins) done in patients undergoing any of the following: minimally invasive aortic, tricuspid, or mitral operations; minimally invasive atrial septal or other structural defect closures; aortic dissection surgery, or cannulation for extracorporeal membrane oxygenation.

In addition, robot-assisted operations for structural heart disease require either vacuum-assisted or kinetic venous drainage, and the perfusion team must be well versed in using this technique.

It is imperative that the lead surgeon be skilled in performing a complete clinical vascular examination. For any patient suspected of having vascular disease, preoperative aortic and aortoiliac computed tomography angiography is recommended before using peripheral cardiopulmonary bypass. In the event of significant descending aortic or peripheral vascular disease, or both, the surgeon must avoid peripheral cannulation and retrograde perfusion for cardiopulmonary bypass.

Using endoballoon aortic occlusion with cardioplegia administration in robotic cardiac surgery requires a specific and complex skill set. Without prior advanced experience in using this technology, alternative clamping techniques should be used during the early phases of this type of program. Only after the cardiac surgery team has attained accomplished endoballoon skills should they be applied to robot-assisted operations.

\section{Additional Requirements}

As stated earlier, robotic cardiac operations should be planned and performed only by a properly assembled team chosen by the lead cardiac surgeons who have had a dedicated interest and demonstrated expertise. To develop a successful robotic program, clinicians with different strengths must be aligned and dedicated to operational success. That requires close collaboration between interventional cardiologists, cardiac surgeons, echocardiographers 
(technicians and cardiologists), cardiac anesthesiologists, perfusionists, operating room personnel, and cardiac intensive care staff. Absence of professional synergy usually leads to poor outcomes and program demise. Moreover, the program must have enthusiastic support from the hospital administration and chief medical officer. Lead surgeons must discuss appropriate patients with other members of their team to select the best treatment algorithm for specific patients.

We would like to stress the importance of a team approach in the operating room. Any of the team members should feel able to call a halt to an operation if the patient is believed to be at risk. Also, team member consistency is very important for robotic cardiac operations, and we recommend that each institution selects one team dedicated to these procedures. New team members should be added only if significant volume warrants it.

Well-organized logistic performance of these operations is crucial to optimizing patient safety. Currently, a hybrid operating room is not required to perform these types of robotic operations. Nevertheless, the hybrid operating room does provide advantages in that high-resolution "catheterization laboratory" imaging capabilities are available in a sterile environment. Using high-resolution X-ray imaging, especially when the endoballoon technology is being used, may facilitate catheter/cannula placement. Moreover, high-resolution imaging is needed for hybrid coronary interventions (robotic surgical with percutaneous coronary stenting) and for postoperative imaging of robot-assisted coronary anastomoses. A minimum operating room requirement is access to an up-to-date $\mathrm{C}$-arm X-ray machine. During a hybrid robotic/percutaneous coronary intervention "same session" procedure, it is advised that one of the following facilities or devices be available: a hybrid operating room, $\mathrm{C}$-arm $\mathrm{X}$-ray equipment, or catheterization laboratories next to operating room facilities. Again, it is absolutely necessary to have TEE available for all intracardiac procedures, ideally with three-dimensional imaging capacity.

\section{Informed Consent}

It is imperative that all available therapeutic alternatives must be conveyed, detailed, and explained to potential robot-assisted surgical patients at consultation as part of the informed consent. These include traditional sternotomy-based, percutaneous, and other minimally invasive methods in addition to the robotic option. The surgeon must thoroughly discuss these different surgical approaches, explaining the different relative risks and benefits of each alternative approach, and proper informed consent should be documented. In addition, the surgeon must discuss the potential for open thoracotomy and sternotomy approaches as potential life-saving measurements should intraoperative technical difficulties arise. That is especially important during early program development. Therefore, with the aim of transparency, we believe the informed consent process should include the robotic team's experience, whether they are being proctored, number of cases performed, and outcomes.

Again, all available therapeutic alternatives must be conveyed and detailed to potential robot-assisted surgical patients at consultation. These include traditional sternotomy-based, percutaneous, and other minimally invasive methods in addition to the robotic option. Again, the surgeon must thoroughly discuss these different surgical approaches, explaining the different relative risks and benefits of each alternative approach, and proper informed consent should be documented.

\section{Program Promoting}

Robotic surgical teams should be very careful in delivering promises to the patient. As mentioned above, the surgeon should also explain "open thoracotomy" or sternotomy approaches as second intraoperative options. The patient should understand that conversion might be life-saving should intraoperative technical difficulties arise. That is especially important during early program development. We cannot overemphasize the importance of obtaining proper informed consent before surgery.

Hospitals and individual surgeons should be careful and circumspect when marketing their programs. Advertising for a robotic program during the implementation phase is discouraged.

\section{PROCEDURAL AND DEVICE-SPECIFIC EDUCATION}

Surgeons initiating a robotic-assisted cardiac surgical program should take advantage of different courses sponsored by The Society of Thoracic Surgeons, The American Association for Thoracic Surgery, European Association for Cardio-Thoracic Surgery, or International Society of Minimally Invasive Cardiac Surgery. These include robotic system as well as procedure-specific training (eg, mitral valve repair, off-pump revascularization, and minimally invasive cardiac surgery approaches). Both formal didactic and hands-on courses help build the basic skills required to perform robotic cardiac operations safely. Training courses should include information regarding the preoperative workup, technical nuances, advantages and disadvantages of the various devices, and the management of intraoperative and postoperative complications. This training should not only be for the operating surgeon; rather, all team members must participate in these training courses. Moreover, the courses should provide opportunities for robust interactions between learning surgeons and experienced leaders.

The surgeon's institution should strongly consider acquiring a virtual simulator or have "wet lab" options to allow surgeons and their teams to practice in a simulation 
environment before their first cases, and then on a regular basis.

Continuation of education should not end with the initial educational course attended by an individual surgeon. To build on the basic knowledge obtained from introductory courses, additional expertise should be obtained through accredited continuing education programs. We suggest that a program should perform at least 20 robotic cardiac surgical procedures per year to maintain proficiency. Individual surgeons are encouraged to participate in specific robotic cardiac surgical continuing education programs.

It is recommended that surgeons performing robotic cardiac surgical operations should consider undergoing additional training. For example, "robotic fellowships" at experienced robotics centers are currently available. The length of these fellowships may be modified in accordance with the minimally invasive cardiac surgical experience of the lead surgeon. These fellowships provide surgeon experience with intracardiac robotic procedures, robotic coronary revascularization, alternative cardiopulmonary perfusion techniques, and diagnostic techniques necessary to perform robotic cardiac operations optimally.

Surgeons and institutions performing these operations should monitor their outcomes through a comprehensive clinical database and make needed changes based on these data. This database can be associated with institutional, state, or professional society national databases. Frequent comprehensive analysis of morbidity and mortality data should be directed toward continual improvement of clinical outcomes, patient safety, and operational process. The position of this task force is that patient safety and quality outcomes must be first priority for every cardiac team performing robot-assisted cardiac surgery and that the patients should receive proper informed consent.

\section{References}

1. Nifong LW, Chu VF, Bailey BM, Maziarz DM, Sorrell VL, Holbert D, et al. Robotic mitral valve repair: experience with the da Vinci system. Ann Thorac Surg. 2003;75:438-43.

2. Nifong LW, Chitwood WR, Pappas PS, Smith CR, Argenziano M, Starnes VA, et al. Robotic mitral valve surgery: a United States multicenter trial. J Thorac Cardiovasc Surg. 2005;129:1395-404.

3. Argenziano M, Katz M, Bonatti J, Srivastava S, Murphy D, Poirier R, et al. Results of the prospective multicenter trial of robotically assisted totally endoscopic coronary artery bypass grafting. Ann Thorac Surg. 2006;81:1666-75.

4. Bonatti J, Schachner T, Bonaros N, Lehr EJ, Zimrin D, Griffith B. Robotically assisted totally endoscopic coronary bypass surgery. Circulation. 2011;124 236-44.

5. Bonaros N, Schachner T, Oehlinger A, Ruetzler E, Kolbitsch C, Dichtl W, et al Robotically assisted totally endoscopic atrial septal defect repair: insights from operative times, learning curves, and clinical outcome. Ann Thorac Surg. 2006;82:687-93

6. Bonatti J, Schachner T, Bonaros N, Oehlinger A, Wiedemann D, Ruetzler E, et al. Effectiveness and safety of total endoscopic left internal mammary artery bypass graft to the left anterior descending artery. Am J Cardiol. 2009;104: 1684-8.

7. Mihaljevic T, Jarrett CM, Gillinov AM, Williams SJ, DeVilliers PA, Stewart WJ, et al. Robotic repair of posterior mitral valve prolapse versus conventional approaches: potential realized. J Thorac Cardiovasc Surg. 2011;141:72-80.

8. Murphy DA, Miller JS, Langford DA. Endoscopic robotic mitral valve surgery. J Thorac Cardiovasc Surg. 2007;133:1119-20.

9. Suri RM, Burkhart HM, Daly RC, Dearani JA, Park SJ, Sundt TM III, et al Robotic mitral valve repair for all prolapse subsets using techniques identical to open valvuloplasty: establishing the benchmark against which percutaneous interventions should be judged. J Thorac Cardiovasc Surg. 2011; 142:970-9.

10. Nifong LW, Rodriguez E, Chitwood WR. 540 consecutive robotic mitral valve repairs including concomitant atrial fibrillation cryoablation. Ann Thorac Surg. 2012;94:38-42.

11. Bonaros N, Schachner T, Lehr E, Kofler M, Wiedemann D, Hong P, et al. Five hundred cases of robotic totally endoscopic coronary artery bypass grafting: predictors of success and safety. Ann Thorac Surg. 2013;95:803-12.

12. Ramzy D, Trento A, Cheng W, De Robertis MA, Mirocha J, Ruzza A, et al. Three hundred robotic-assisted mitral repairs: the Cedars-Sinai experience. J Thorac Cardiovasc Surg. 2014;147:228-35.

13. Halkos ME, Liberman HA, Devireddy C, Walker P, Finn AV, Jaber W, et al. Early clinical and angiographic outcomes after robotic-assisted coronary artery bypass surgery. J Thorac Cardiovasc Surg. 2014;147:179-85.

14. Wang S, Zhou J, Cai F. Traditional coronary artery bypass graft versus totally endoscopic coronary artery bypass graft or robot-assisted coronary artery bypass graft—meta-analysis of 16 studies. Eur Rev Med Pharmacol Sci. 2014;18:790-7. 